\title{
Concomitant Papillary Thyroid Cancer and Graves' disease: An ominous association
}

\author{
Ryanputra $D^{1}$, Seow $C J^{2}$
}

${ }^{1}$ Yong Loo Lin School of Medicine, National University of Singapore, Singapore

${ }^{2}$ Department of Endocrinology, Tan Tock Seng Hospital, Singapore

\section{Background}

Papillary thyroid carcinoma (PTC) in patients with background Graves' disease (GD) has been associated with a higher risk of post-operative tumour recurrence. We present a case and review the literature for possible explanations.

\section{Case Presentation}

A 54 year-old Chinese gentleman with background history of hypertension was first diagnosec with Graves' disease when he presented to the polyclinic for weight loss, heat intolerance ano palpitations of 2 to 3 months duration. His thyroid function test showed a free thyroxine (fT4) level that is elevated at $\mathbf{6 7 . 5} \mathrm{pmol} / \mathrm{L}$ (RI: 11.8-24.6), a TSH level suppressed at $0.006 \mathrm{IU} / \mathrm{L}$ (RI: 0.27-4.20) and a TSH receptor antibody (TRAb) level of $6.2 \mathrm{IU} / \mathrm{L}$. He was started on carbimazole with normalization of his thyroid function within 6 months of treatment.

The patient was referred to the Endocrinology clinic a year later in 2011 for a solitary nodule which was growing in size. There were no other nodules, and further history obtained did not suggest increased likelihood of malignancy. He did not have any head and neck radiation and there were no family history of malignancies. He was not on any long term medications except for carbimazole and has no known drug allergies. He does not smoke or drink alcohol. There wa no family history of thyroid disorders. Physical examination revealed a diffusely enlarged goiter with a palpable right sided thyroid nodule. There was no cervical lymphadenopathy. The rest of the examination was unremarkable.

His repeated thyroid function tests were normal with fT4 of $10 \mathrm{pmol} / \mathrm{L}$ and a TSH of $2.40 \mathrm{mIU} / \mathrm{L}$ The TRAb was detectable at $3.6 \mathrm{IU} / \mathrm{L}$. An ultrasound of the thyroid gland (Figure 1) was performed and showed a $3.4 \times 2.4 \times 2.5 \mathrm{~cm}$ isoechoic nodule with peripheral halo and vascularity in the lower pole of the right lobe. In addition, there was a similar $0.9 \times 0.7 \times 0.2 \mathrm{~cm}$ lesion notec in the upper pole of the left lobe. There were no lymph nodes detected. Fine needle aspiration cytology (FNAC) showed follicular epithelial cells demonstrating dense cytoplasm, occasional grooves and easily identifiable nuclear inclusion, which were highly suspicious for papillary thyroid cancer.

A total thyroidectomy with prophylactic central-compartment neck dissection was arranged the following week. The surgically resected specimen showed a $4.7 \times 2 \times 2 \mathrm{~cm}$ nodule in the right upper lobe as well as a $0.5 \mathrm{~cm}$ nodule adjacent to it. In addition, there were 2 adjacent nodules within the isthmus and pyramidal lobes measuring $2 \times 0.5 \times 0.5 \mathrm{~cm}$ and $1 \times 0.5 \times 0.5 \mathrm{~cm}$ respectively. The right culprit nodule showed classical features of papillary thyroid carcinoma with minimal capsular invasion. (Figure 2) The rest of the nodules and the level 6 lymph nodes do not exhibit features of malignancy. Based on the TNM classification system for differentiated thyroid carcinoma, he was classified as Stage III (T3NOMO). He subsequently received $150 \mathrm{mCi}$ of radioactive iodine. A I-131 whole body scan performed 48 hours after RAl ablation therapy showed very mild focal I-131 uptake at the thyroid bed, with 48 -hour uptake of $0.09 \%$, likely representing remnant thyroid tissue. Whole body survey reveals no abnormal focal uptake of 1131 elsewhere.

The patient was initiated on TSH suppressive therapy with levothyroxine (LT4) replacement complemented by an annual ultrasound of the thyroid. 3 years later, a right mid-cervical lymph node was found on ultrasound to be enlarged at $1.5 \times 0.8 \times 0.8 \mathrm{~cm}$ with loss of fatty hilum. (Figure 3) TRAb level was elevated at 3.3 IU/L (RI: 0-1.0) Subsequent FNAC showed clusters of epithelial cells with enlarged, hyperchromatic and irregular nuclei, anisonucleosis, nuclear grooves and nuclear pseudoinclusions consistent with lymph node recurrence. MRI of the neck showed a $8 \mathrm{~mm}$ right level III lymph node, appearing to correspond with the lobulated lymph node seen on the ultrasound. (Figure 4) A prominent 9mm right level I B node and a small cluster of lymph nodes is also noted in the left supraclavicular fossa, the largest measuring $10 \mathrm{~mm}$ in diameter.

The patient subsequently underwent right modified radical neck dissection and received another $150 \mathrm{mCi}$ of radioactive iodine after surgery. He has also been counseled for external beam radiotherapy.

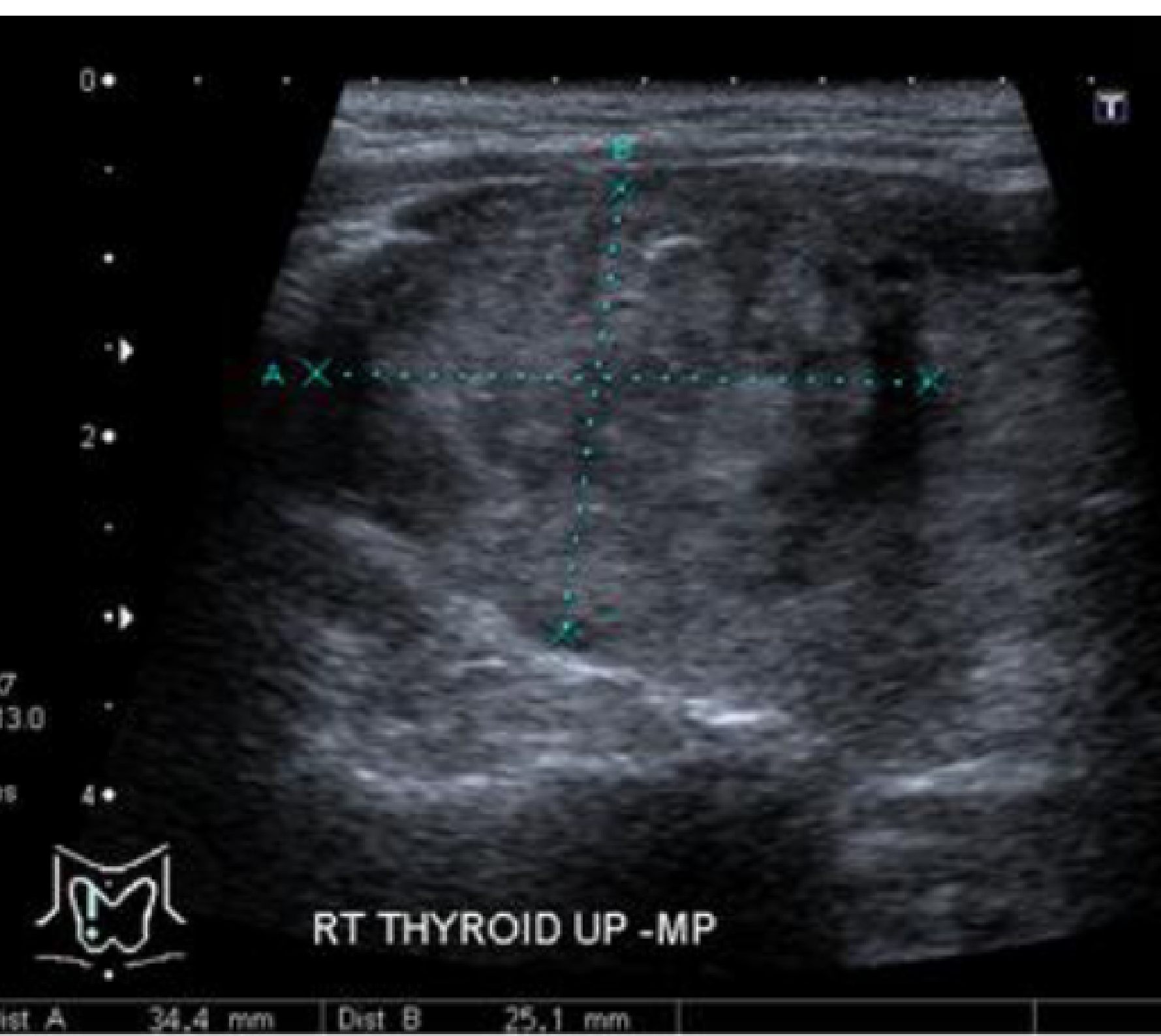

Figure 1: Thyroid ultrasound on initial presentation

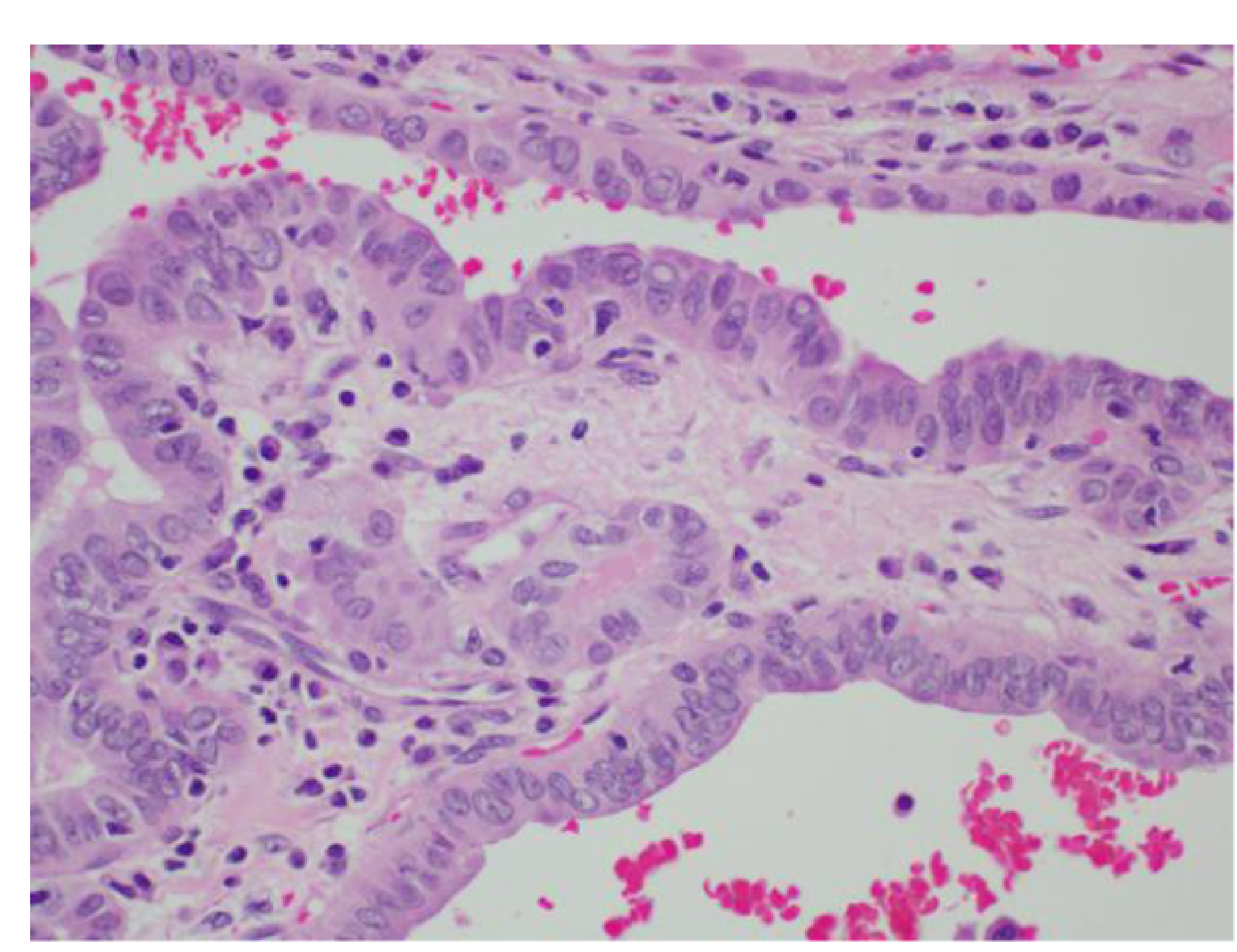

Figure 2: Histology of specimen showing papillary thyroid carcinoma

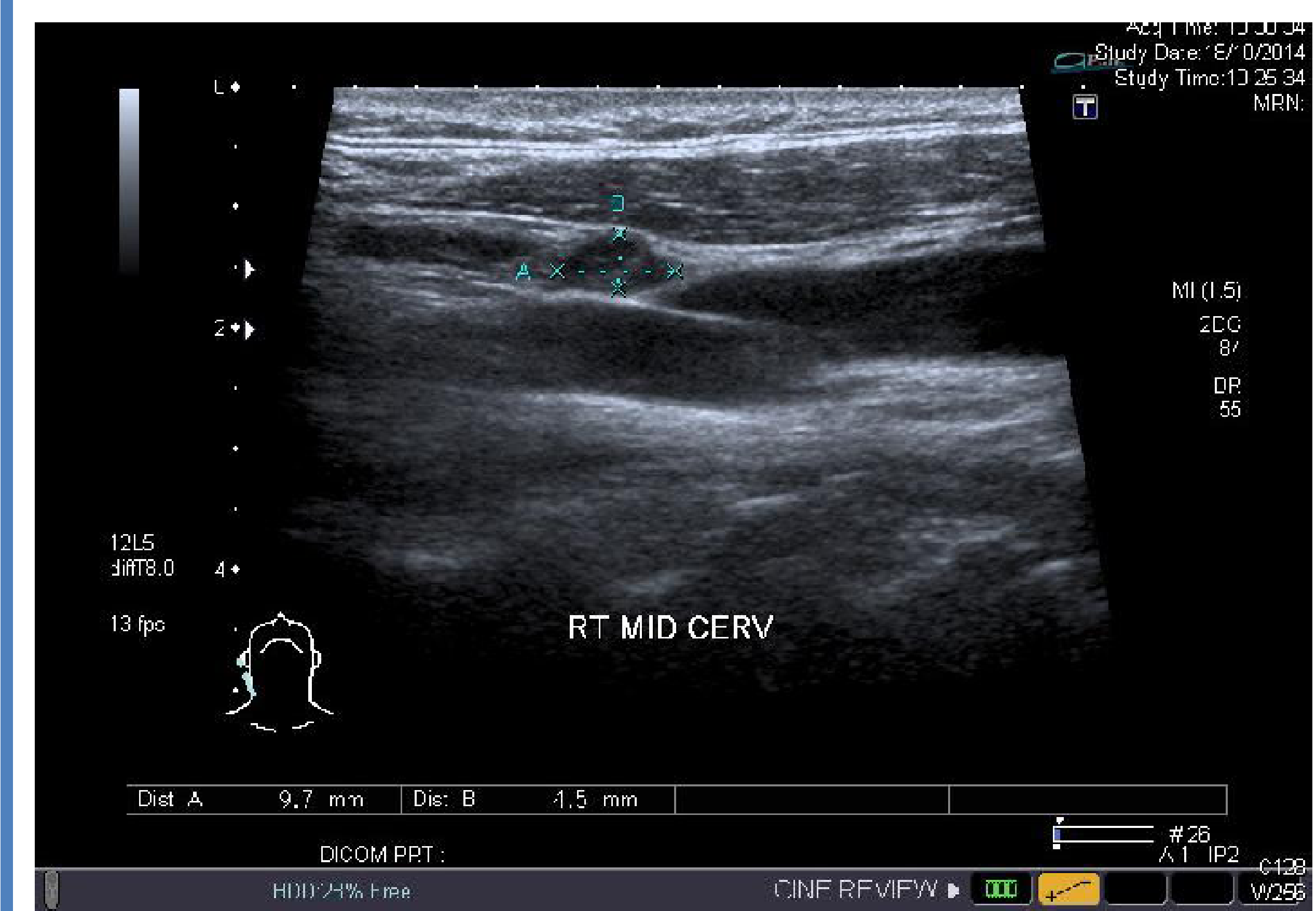

Figure 3: Thyroid ultrasound on follow-up

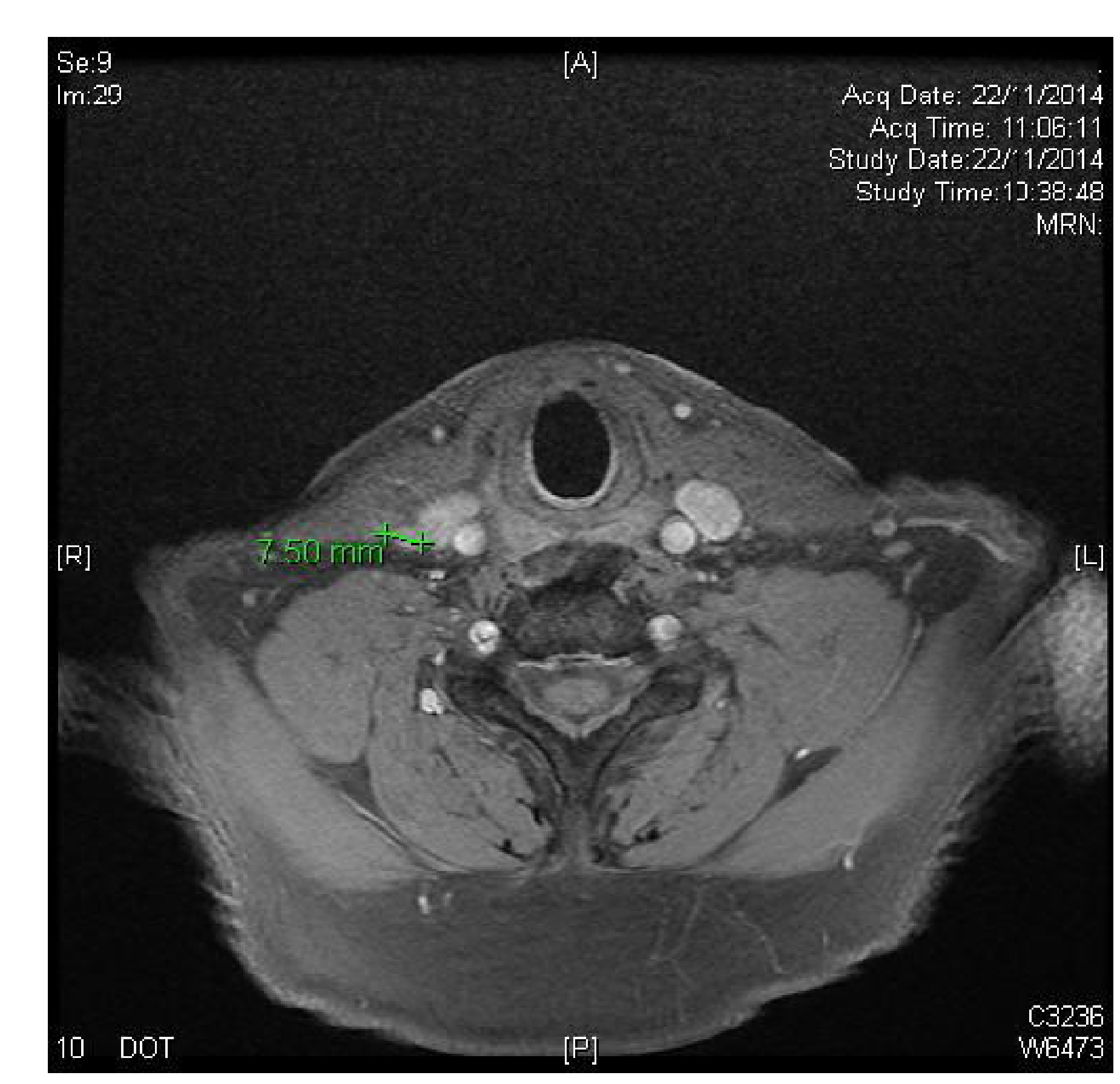

Figure 4: Neck MRI showing lymph node

\section{Discussion}

Palpable thyroid nodules occur in approximately $15 \%$ of patients with GD compared with the usual rate of $5 \%$ observed in the general population. The malignancy rate ranges from $10 \%$ to $46 \%$, somewhat higher than that observed in euthyroid patients. $(1,2)$ The incidence of PTC has been shown to be higher in patients with GD than patients without GD (3).

The outcome of thyroid carcinoma concurrent to GD is mixed, however, with some studies observing an increase in metastases to regional lymph nodes (4) and mortality (1) while others reporting no significant differences in multifocality, lymph node metastases, distant metastases or mortality compared to that of euthyroid patients matched for age. (5-7) Aggressive variants of PTC such as the tall cell, columnar and insular types, nevertheless, have been linked to higher risks of recurrence. (8) One study suggested an increased incidence of the tall cell variant (TCV) in patients with nodular GD, presenting an increased risk of PTC recurrence; however, there was no significant difference in overall recurrence rates between patients with and without GD within that study cohort (3). The possible reasons that could explain the increased frequency and aggressiveness of clinical thyroid cancer reported by some studies for patients with GD are not clear. It should be noted that some papers compare outcomes between patients with GD who underwent surgery for hyperthyroidism (with more incidental micro-PTCs), against patients without GD who underwent surgery for palpable nodules (with more aggressive PTCs) (9), hence possibly underestimating the incidence of poorer outcomes for malignancies in patients with GD.

Neoplastic cells of differentiated thyroid cancer, like normal thyroid cells, express functional receptors for TSH. In GD, antibodies produced have strong, agonistic activity to the TSH-Receptor (TSHR); this resulted in antibody-mediated stimulation of the receptor. Stimulation of TSHR by antibodies leads to secretion of thyroid hormone and hyperthyroidism independently of the Hypothalamus-Pituitary-Thyroid (HPT) axis. Moreover, thyroid stimulating antibodies might play a role in stimulating thyroid cancer growth, invasiveness (10) and angiogenesis by upregulating vascular endothelial growth factor, placenta growth factor, and their receptors. Stimulating TSHR antibodies have also been shown to initiate cellular proliferation by suppressing mitochondrial reactive oxygen species levels, preventing apoptosis (11). Apart from that, different growth factors that probably are produced by the over-stimulated (by TRAbs) and hypervascularised thyroid ( 3 ) could also affect the growth and metastases of thyroid cancer in patients with GD. In addition to the effect of stimulating TSHR-Abs, interleukins 4 and 10, locally produced in thyroid glands affected by $\mathrm{GD}$, also have a strong anti-apoptotic effect on malignant thyrocytes (12). These factors may contribute to thyroid cancer progression and unfavourable prognosis in patients with GD. Given the age, size of the tumor, extrathyroidal extension (albeit minimal) as well as the background of GD (13), it will be reasonable to label him as being of intermediate risk of recurrence.

\section{Conclusion}

Given that Graves' disease is potentially associated with a higher risk of recurrence for papillary thyroid carcinoma, one needs to have a heightened awareness and be more vigilant in monitoring patients for disease recurrence.

\section{References}

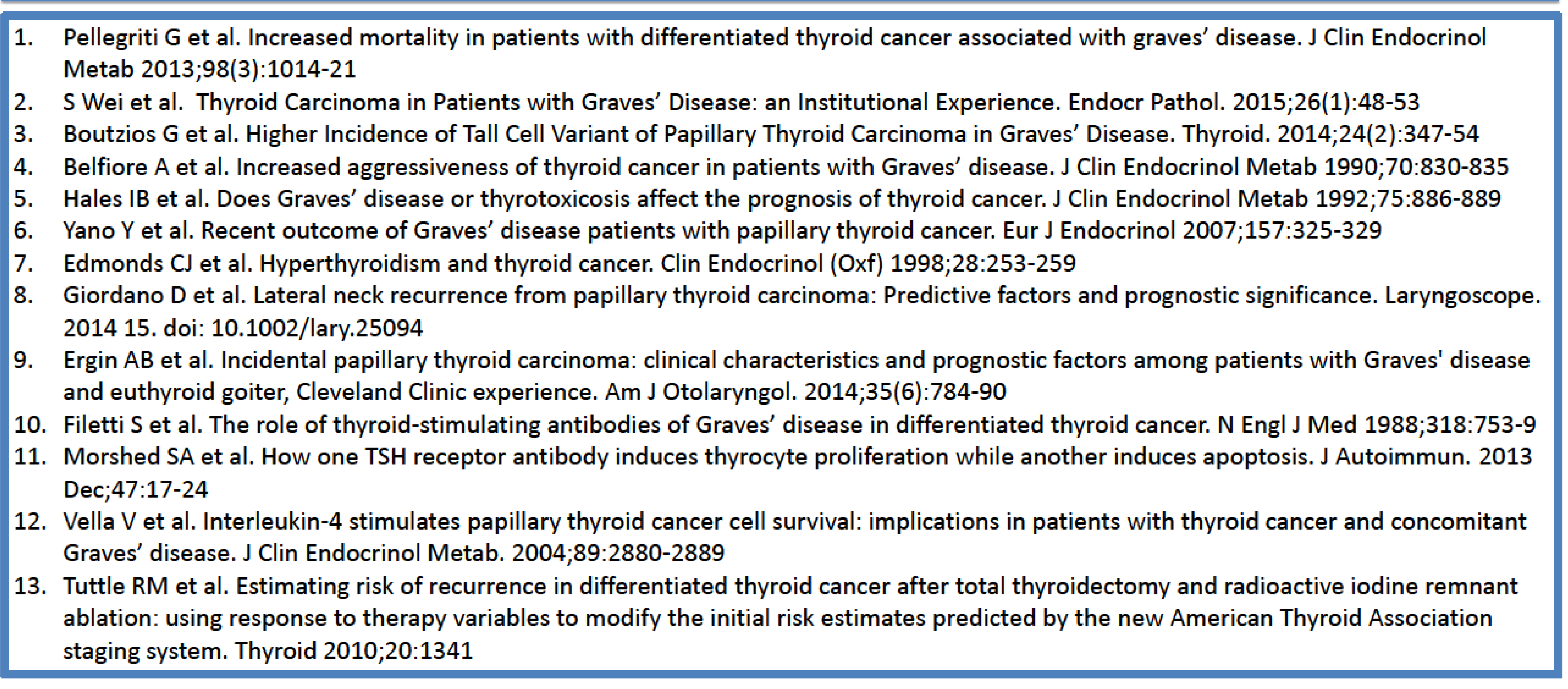

